\title{
A new generalization of Ostrowski-Grüss type inequalities involving functions of two independent variables
}

Qiaoling Xue, Shunfeng Wang, and Wenjun Liu 


\title{
A NEW GENERALIZATION OF OSTROWSKI-GRÜSS TYPE INEQUALITIES INVOLVING FUNCTIONS OF TWO INDEPENDENT VARIABLES
}

\author{
QIAOLING XUE, SHUNFENG WANG, AND WENJUN LIU
}

Received March 30, 2010

Abstract. The main purpose of this paper is to derive a new inequality of Ostrowski-Grüss type with a parameter involving functions of two independent variables.

2000 Mathematics Subject Classification: 26D15

Keywords: Ostrowski-Grüss type inequality, functions of two independent variables

\section{INTRODUCTION}

In [4], Dragomir and Wang proved the following Ostrowski-Grüss type integral inequality.

Theorem 1. Let $f:[a, b] \rightarrow R$ be continuous on $[a, b]$, differentiable on $(a, b)$ and suppose that $\gamma \leq f^{\prime}(x) \leq \Gamma$ for all $x \in(a, b)$. Then we have

$$
\left|f(x)-\frac{f(b)-f(a)}{b-a}\left(x-\frac{a+b}{2}\right)-\frac{1}{b-a} \int_{a}^{b} f(t) d t\right| \leq \frac{1}{4}(b-a)(\Gamma-\gamma)
$$

for all $x \in[a, b]$.

In [2], Cheng not only gave a sharp version of the above inequality but also generalized it as follows.

Theorem 2. Let the assumptions of Theorem 1 hold. Then for all $x \in[a, b]$, we have

$\left|\frac{1}{2} f(x)-\frac{(x-b) f(b)-(x-a) f(a)}{2(b-a)}-\frac{1}{b-a} \int_{a}^{b} f(t) d t\right| \leq \frac{1}{2} \frac{(x-a)^{2}+(b-x)^{2}}{2(b-a)} \frac{\Gamma-\gamma}{2}$.

In a recent paper [12], Wang et al. proved the following inequality with a parameter.

This paper was typeset using $\mathcal{A M}_{\mathcal{M}} \mathcal{S}$-LATEX. 
Theorem 3. Let the assumptions of Theorem 1 hold. Then for all $x \in[a, b]$ and $\lambda \in[0,1]$, we have

$$
\begin{aligned}
& \mid\left(1-\frac{\lambda}{2}\right) f(x)-\lambda \frac{(x-b) f(b)-(x-a) f(a)}{2(b-a)} \\
& -\frac{\Gamma+\gamma}{2}(1-\lambda)\left(x-\frac{a+b}{2}\right)-\frac{1}{b-a} \int_{a}^{b} f(t) d t \mid \\
& \leq\left(1-\lambda+\frac{\lambda^{2}}{2}\right) \frac{(x-a)^{2}+(b-x)^{2}}{2(b-a)} \frac{\Gamma-\gamma}{2} .
\end{aligned}
$$

More recently, Sarikaya [10] established the following generalization of (1.2) involving functions of two independent variables.

Theorem 4. Let $f:[a, b] \times[c, d] \rightarrow R$ be an absolutely continuous function such that the partial derivative of order 2 exist and suppose that there exist constants $\gamma, \Gamma \in R$ with $\gamma \leq \frac{\partial^{2} f(t, s)}{\partial t \partial s} \leq \Gamma$ for all $(t, s) \in[a, b] \times[c, d]$. Then we have

$$
\begin{aligned}
& \mid \frac{1}{4} f(x, y)+\frac{1}{4} H(x, y)-\frac{1}{2(b-a)} \int_{a}^{b} f(t, y) d t-\frac{1}{2(d-c)} \int_{c}^{d} f(x, s) d s \\
& -\frac{1}{2(b-a)(d-c)}\left\{\int_{a}^{b}[(y-c) f(t, c)+(d-y) f(t, d)] d t\right. \\
& \left.\quad+\int_{c}^{d}[(x-a) f(a, s)+(b-x) f(b, s)] d s\right\} \\
& +\frac{1}{(b-a)(d-c)} \int_{a}^{b} \int_{c}^{d} f(t, s) d t d s \mid \\
& \leq \frac{1}{4} \frac{\left[(x-a)^{2}+(b-x)^{2}\right]\left[(y-c)^{2}+(d-y)^{2}\right]}{4(b-a)(d-c)} \frac{\Gamma-\gamma}{2}
\end{aligned}
$$

for all $(x, y) \in[a, b] \times[c, d]$, where

$$
\begin{gathered}
H(x, y)= \\
\frac{(x-a)[(y-c) f(a, c)+(d-y) f(a, d)]+(b-x)[(y-c) f(b, c)+(d-y) f(b, d)]}{(b-a)(d-c)} \\
+\frac{(x-a) f(a, y)+(b-x) f(b, y)}{b-a}+\frac{(y-c) f(x, c)+(d-y) f(x, d)}{d-c} .
\end{gathered}
$$

For other related work, we refer the reader to [3, 5-9, 12].

In this paper, we shall derive a new inequality of Ostrowski-Grüss type with a parameter for absolutely continuous functions of two independent variables, which will not only provides a generalization of the inequalities (1.4) and (1.5), but also gives some other interesting inequalities as special cases. 


\section{MAIN RESULT}

Theorem 5. Let $f:[a, b] \times[c, d] \rightarrow R$ be an absolutely continuous function such that the partial derivative of order 2 exist and suppose that there exist constants $\gamma, \Gamma \in R$ with $\gamma \leq \frac{\partial^{2} f(t, s)}{\partial t \partial s} \leq \Gamma$ for all $(t, s) \in[a, b] \times[c, d]$. Then we have

$$
\begin{gathered}
\mid\left(1-\frac{\lambda}{2}\right)^{2} f(x, y)+\frac{\lambda}{2}\left(1-\frac{\lambda}{2}\right) H_{1}(x, y)+\left(\frac{\lambda}{2}\right)^{2} H_{2}(x, y) \\
-\left(1-\frac{\lambda}{2}\right)\left[\frac{1}{b-a} \int_{a}^{b} f(t, y) d t+\frac{1}{d-c} \int_{c}^{d} f(x, s) d s\right] \\
-\frac{\lambda}{2} \frac{1}{(b-a)(d-c)}\left\{\int_{a}^{b}[(y-c) f(t, c)+(d-y) f(t, d)] d t\right. \\
\left.\quad+\int_{c}^{d}[(x-a) f(a, s)+(b-x) f(b, s)] d s\right\} \\
-\frac{\Gamma+\gamma}{2}(1-\lambda)^{2}\left(x-\frac{a+b}{2}\right)\left(y-\frac{c+d}{2}\right)+\frac{1}{(b-a)(d-c)} \int_{a}^{b} \int_{c}^{d} f(t, s) d t d s \mid \\
\leq\left(1-\lambda+\frac{\lambda^{2}}{2}\right)^{2} \frac{\left[(x-a)^{2}+(b-x)^{2}\right]\left[(y-c)^{2}+(d-y)^{2}\right]}{4(b-a)(d-c)} \frac{\Gamma-\gamma}{2} \quad(2.1)
\end{gathered}
$$

for all $(x, y) \in[a, b] \times[c, d]$ and $\lambda \in[0,1]$, where

$$
H_{1}(x, y)=\frac{(x-a) f(a, y)+(b-x) f(b, y)}{b-a}+\frac{(y-c) f(x, c)+(d-y) f(x, d)}{d-c}
$$

and

$$
=\frac{(x-a)[(y-c) f(a, c)+(d-y) f(a, d)]+(b-x)[(y-c) f(b, c)+(d-y) f(b, d)]}{(b-a)(d-c)} .
$$

Proof. We define the mapping: $p:[a, b] \times[a, b] \rightarrow R, q:[c, d] \times[c, d] \rightarrow R$ as

$$
p(x, t)= \begin{cases}t-\left(a+\lambda \frac{x-a}{2}\right) & t \in[a, x] \\ t-\left(b-\lambda \frac{b-x}{2}\right) & t \in(x, b]\end{cases}
$$

and

$$
q(y, s)= \begin{cases}s-\left(c+\lambda \frac{y-c}{2}\right) & s \in[c, y] \\ s-\left(d-\lambda \frac{d-y}{2}\right) & s \in(y, d]\end{cases}
$$


By definitions of $p(x, t)$ and $q(y, s)$, we have

$$
\begin{gathered}
\int_{a}^{b} \int_{c}^{d} p(x, t) q(y, s) \frac{\partial^{2} f(t, s)}{\partial t \partial s} d s d t \\
=\int_{a}^{x} \int_{c}^{y}\left[t-\left(a+\lambda \frac{x-a}{2}\right)\right]\left[s-\left(c+\lambda \frac{y-c}{2}\right)\right] \frac{\partial^{2} f(t, s)}{\partial t \partial s} d s d t \\
+\int_{a}^{x} \int_{y}^{d}\left[t-\left(a+\lambda \frac{x-a}{2}\right)\right]\left[s-\left(d-\lambda \frac{d-y}{2}\right)\right] \frac{\partial^{2} f(t, s)}{\partial t \partial s} d s d t \\
+\int_{x}^{b} \int_{c}^{y}\left[t-\left(b-\lambda \frac{b-x}{2}\right)\right]\left[s-\left(c+\lambda \frac{y-c}{2}\right)\right] \frac{\partial^{2} f(t, s)}{\partial t \partial s} d s d t \\
+\int_{x}^{b} \int_{y}^{d}\left[t-\left(b-\lambda \frac{b-x}{2}\right)\right]\left[s-\left(d-\lambda \frac{d-y}{2}\right)\right] \frac{\partial^{2} f(t, s)}{\partial t \partial s} d s d t
\end{gathered}
$$

Integrating by parts twice, we can state:

$$
\begin{gathered}
\int_{a}^{x} \int_{c}^{y}\left[t-\left(a+\lambda \frac{x-a}{2}\right)\right]\left[s-\left(c+\lambda \frac{y-c}{2}\right)\right] \frac{\partial^{2} f(t, s)}{\partial t \partial s} d s d t \\
=(x-a)(y-c)\left[\left(1-\frac{\lambda}{2}\right)^{2} f(x, y)+\left(1-\frac{\lambda}{2}\right) \frac{\lambda}{2} f(a, y)\right. \\
\left.+\left(1-\frac{\lambda}{2}\right) \frac{\lambda}{2} f(x, c)+\left(\frac{\lambda}{2}\right)^{2} f(a, c)\right] \\
-(y-c) \int_{a}^{x}\left[\left(1-\frac{\lambda}{2}\right) f(t, y)+\frac{\lambda}{2} f(t, c)\right] d t \\
-(x-a) \int_{c}^{y}\left[\left(1-\frac{\lambda}{2}\right) f(x, s)+\frac{\lambda}{2} f(a, s)\right] d s+\int_{a}^{x} \int_{c}^{y} f(t, s) d s d t \\
\int_{a}^{x} \int_{y}^{d}\left[t-\left(a+\lambda \frac{x-a}{2}\right)\right]\left[s-\left(d-\lambda \frac{d-y}{2}\right)\right] \frac{\partial^{2} f(t, s)}{\partial t \partial s} d s d t \\
{\left[\left(1-\frac{\lambda}{2}\right)^{2} f(x, y)+\left(1-\frac{\lambda}{2}\right) \frac{\lambda}{2} f(a, y)+\left(1-\frac{\lambda}{2}\right) \frac{\lambda}{2} f(x, d)+\left(\frac{\lambda}{2}\right)^{2} f(a, d)\right]} \\
-(d-y) \int_{a}^{x}\left[\left(1-\frac{\lambda}{2}\right) f(t, y)+\frac{\lambda}{2} f(t, d)\right] d t \\
-(x-a) \int_{y}^{d}\left[\left(1-\frac{\lambda}{2}\right) f(x, s)+\frac{\lambda}{2} f(a, s)\right] d s+\int_{a}^{x} \int_{y}^{d} f(t, s) d s d t
\end{gathered}
$$




$$
\begin{gathered}
\int_{x}^{b} \int_{c}^{y}\left[t-\left(b-\lambda \frac{b-x}{2}\right)\right]\left[s-\left(c+\lambda \frac{y-c}{2}\right)\right] \frac{\partial^{2} f(t, s)}{\partial t \partial s} d s d t \\
=(b-x)(y-c) \\
{\left[\left(1-\frac{\lambda}{2}\right)^{2} f(x, y)+\left(1-\frac{\lambda}{2}\right) \frac{\lambda}{2} f(b, y)+\left(1-\frac{\lambda}{2}\right) \frac{\lambda}{2} f(x, c)+\left(\frac{\lambda}{2}\right)^{2} f(b, c)\right]} \\
-(y-c) \int_{x}^{b}\left[\left(1-\frac{\lambda}{2}\right) f(t, y)+\frac{\lambda}{2} f(t, c)\right] d t \\
-(b-x) \int_{c}^{y}\left[\left(1-\frac{\lambda}{2}\right) f(x, s)+\frac{\lambda}{2} f(b, s)\right] d s+\int_{x}^{b} \int_{c}^{y} f(t, s) d s d t, \\
\int_{x}^{b} \int_{y}^{b}\left[t-\left(b-\lambda \frac{b-x}{2}\right)\right]\left[s-\left(c-\lambda \frac{d-y}{2}\right)\right]^{2} \frac{\partial^{2} f(t, s)}{\partial t \partial s} d s d t \\
{\left[\left(1-\frac{\lambda}{2}\right)^{2} f(x, y)+\left(1-\frac{\lambda}{2}\right) \frac{\lambda}{2} f(b, y)+\left(1-\frac{\lambda}{2}\right) \frac{\lambda}{2} f(x, d)+\left(\frac{\lambda}{2}\right)^{2} f(b, d)\right]} \\
-(d-y) \int_{x}^{b}\left[\left(1-\frac{\lambda}{2}\right) f(t, y)+\frac{\lambda}{2} f(t, d)\right] d t \\
-(b-x) \int_{c}^{y}\left[\left(1-\frac{\lambda}{2}\right) f(x, s)+\frac{\lambda}{2} f(b, s)\right] d s+\int_{x}^{b} \int_{y}^{d} f(t, s) d s d t .
\end{gathered}
$$

After adding (2)-(2) and rewriting we easily deduce:

$$
\begin{gathered}
\int_{a}^{b} \int_{c}^{d} p(x, t) q(y, s) \frac{\partial^{2} f(t, s)}{\partial t \partial s} d s d t \\
=(b-a)(d-c)\left(1-\frac{\lambda}{2}\right)^{2} f(x, y) \\
+\frac{\lambda}{2}\left(1-\frac{\lambda}{2}\right)\{(b-a)[(y-c) f(x, c)+(d-y) f(x, d)] \\
+(d-c)[(x-a) f(a, y)+(b-x) f(b, y)]\} \\
+\left(\frac{\lambda}{2}\right)^{2}\{(x-a)[(y-c) f(a, c)+(d-y) f(a, d)] \\
+(b-x)[(y-c) f(b, c)+(d-y) f(b, d)]\} \\
-\frac{\lambda}{2}\left\{\int_{a}^{b}[(y-c) f(t, c)+(d-y) f(t, d)] d t+\int_{c}^{d}[(x-a) f(a, s)+(b-x) f(b, s)] d s\right\}
\end{gathered}
$$




$$
-\left(1-\frac{\lambda}{2}\right)\left[(d-c) \int_{a}^{b} f(t, y) d t+(b-a) \int_{c}^{d} f(x, s) d s\right]+\int_{a}^{b} \int_{c}^{d} f(s, t) d s d t .
$$

We also have

$$
\int_{a}^{b} \int_{c}^{d} p(x, t) q(y, s) d s d t=(b-a)(d-c)(1-\lambda)^{2}\left(x-\frac{a+b}{2}\right)\left(y-\frac{c+d}{2}\right)
$$

Let $M=\frac{\Gamma+\gamma}{2}$. From (2) and (2.8), it follows that

$$
\begin{gathered}
\int_{a}^{b} \int_{c}^{d} p(x, t) q(y, s)\left[\frac{\partial^{2} f(t, s)}{\partial t \partial s}-M\right] d s d t \\
=\int_{a}^{b} \int_{c}^{d} p(x, t) q(y, s) \frac{\partial^{2} f(t, s)}{\partial t \partial s} d t d s \\
-M(b-a)(d-c)(1-\lambda)^{2}\left(x-\frac{a+b}{2}\right)\left(y-\frac{c+d}{2}\right) .
\end{gathered}
$$

On the other hand, we get

$$
\begin{array}{r}
\left|\int_{a}^{b} \int_{c}^{d} p(x, t) q(y, s)\left[\frac{\partial^{2} f(t, s)}{\partial t \partial s}-M\right] d s d t\right| \\
\leq \max _{(t, s) \in[a, b] \times[c, d]}\left|\frac{\partial^{2} f(t, s)}{\partial t \partial s}-M\right| \int_{a}^{b} \int_{c}^{d}|p(x, t) q(y, s)| d t d s .
\end{array}
$$

We also have$$
\max _{(t, s) \in[a, b] \times[c, d]}\left|\frac{\partial^{2} f(t, s)}{\partial t \partial s}-M\right| \leq \frac{\Gamma-\gamma}{2}
$$

and

$$
\begin{gathered}
\int_{a}^{b} \int_{c}^{d}|p(x, t) q(y, s)| d s d t \\
=\left(1-\lambda+\frac{\lambda^{2}}{2}\right)^{2} \frac{\left[(x-a)^{2}+(b-x)^{2}\right]\left[(y-c)^{2}+(d-y)^{2}\right]}{4}
\end{gathered}
$$

From (2) to (2), we easily get

$$
\begin{gathered}
\left|\int_{a}^{b} \int_{c}^{d} p(x, t) q(y, s)\left[\frac{\partial^{2} f(t, s)}{\partial t \partial s}-M\right] d s d t\right| \\
\leq\left(1-\lambda+\frac{\lambda^{2}}{2}\right)^{2} \frac{\left[(x-a)^{2}+(b-x)^{2}\right]\left[(y-c)^{2}+(d-y)^{2}\right]}{4} \frac{\Gamma-\lambda}{2} .
\end{gathered}
$$

From (2), (2) and (2), we see that (5) holds. 
Corollary 1. Under the assumptions of Theorem 5, we have

$$
\begin{aligned}
& \mid f(x, y)-\frac{1}{b-a} \int_{a}^{b} f(t, y) d t-\frac{1}{d-c} \int_{c}^{d} f(x, s) d s \\
& -\frac{\Gamma+\gamma}{2}\left(x-\frac{a+b}{2}\right)\left(y-\frac{c+d}{2}\right)+\frac{1}{(b-a)(d-c)} \int_{a}^{b} \int_{c}^{d} f(t, s) d t d s \mid \\
& \leq \frac{\left[(x-a)^{2}+(b-x)^{2}\right]\left[(y-c)^{2}+(d-y)^{2}\right]}{8(b-a)(d-c)}(\Gamma-\gamma),
\end{aligned}
$$

and especially

$$
\begin{gathered}
\mid f\left(\frac{a+b}{2}, \frac{c+d}{2}\right)-\frac{1}{b-a} \int_{a}^{b} f\left(t, \frac{c+d}{2}\right) d t \\
-\frac{1}{d-c} \int_{c}^{d} f\left(\frac{a+b}{2}, s\right) d s+\frac{1}{(b-a)(d-c)} \int_{a}^{b} \int_{c}^{d} f(t, s) d t d s \mid \\
\leq(\Gamma-\gamma) \frac{(b-a)(d-c)}{16} .
\end{gathered}
$$

Proof. We take $\lambda=0$ in (5) to get (2.14) and $x=\frac{a+b}{2}, y=\frac{c+d}{2}$ in (2.14) to get (1).

Remark 1. If we assume that $f(s, t)=h(s) h(t), h:[a, b] \rightarrow R$, then from (5) we can get (1.4) (for $x=y$ ). Consequently, (5) can be also regarded as a generalization of (1.4) for double integrals. If we take $\lambda=1$ in (5), then the inequality (1.5) is recaptured. Thus (5) may also be regarded as a generalization of (1.5) with a parameter.

Remark 2. If we take $\lambda=\frac{1}{2}$, or $\lambda=\frac{1}{3}$, or $x=\frac{a+b}{2}$ and $y=\frac{c+d}{2}$, or $x=a$ and $y=c$, or $x=a$ and $y=d$, or $x=b$ and $y=c$, or $x=b$ and $y=d$ in (5), we can get other interesting inequalities for these special cases.

Remark 3. As in $[1,4,11]$, we can apply the above obtained inequalities (5) et al. in numerical integration obtaining some general cubature formulae.

\section{ACKNOWLEDGMENTS}

The work was supported by the Science Research Foundation of Nanjing University of Information Science and Technology (Grant No. 20070088) and the Natural Science Foundation of the Jiangsu Higher Education Institutions (Grant No. 09KJB110005).

\section{REFERENCES}

[1] N. S. Barnett and S. S. Dragomir, "An Ostrowski type inequality for double integrals and applications for cubature formulae,” Soochow J. Math., vol. 27, no. 1, pp. 1-10, 2001. 
[2] X.-L. Cheng, "Improvement of some Ostrowski-Grüss type inequalities," Comput. Math. Appl., vol. 42, no. 1-2, pp. 109-114, 2001.

[3] S. S. Dragomir, P. Cerone, and J. Roumeliotis, "A new generalization of Ostrowski's integral inequality for mappings whose derivatives are bounded and applications in numerical integration and for special means," Appl. Math. Lett., vol. 13, no. 1, pp. 19-25, 2000.

[4] S. S. Dragomir and S. Wang, "An inequality of Ostrowski-Grüss type and its applications to the estimation of error bounds for some special means and for some numerical quadrature rules," Comput. Math. Appl., vol. 33, no. 11, pp. 15-20, 1997.

[5] W. Liu, "Several error inequalities for a quadrature formula with a parameter and applications," Comput. Math. Appl., vol. 56, no. 7, pp. 1766-1772, 2008.

[6] W. Liu, Q.-L. Xue, and S.-F. Wang, "Several new perturbed Ostrowski-like type inequalities," JIPAM. J. Inequal. Pure Appl. Math., vol. 8, no. 4, pp. Article 110, 6, 2007.

[7] W. J. Liu, Q.-A. Ngo, and W. B. Chen, "A new generalization of Ostrowski type inequality on time scales," An. Ştiinț. Univ. "Ovidius" Constanţa Ser. Mat., vol. 17, no. 2, pp. 101-114, 2009.

[8] Z. Liu, "Note on a paper by N. Ujević: "Sharp inequalities of Simpson type and Ostrowski type" [Comput. Math. Appl. 48 (2004), no. 1-2, 145-151; MR2086792]," Appl. Math. Lett., vol. 20, no. 6, pp. 659-663, 2007.

[9] Z. Lü, "On sharp inequalities of Simpson type and Ostrowski type in two independent variables," Comput. Math. Appl., vol. 56, no. 8, pp. 2043-2047, 2008.

[10] M. Z. Sarikaya, "On the Ostrowski type integral inequality," Acta Math. Univ. Comenian. (N.S.), vol. 79, no. 1, pp. 129-134, 2010.

[11] N. Ujević, "A generalization of Ostrowski's inequality and applications in numerical integration," Appl. Math. Lett., vol. 17, no. 2, pp. 133-137, 2004.

[12] S.-F. Wang, Q.-L. Xue, and W.-J. Liu, "Further generalization of Ostrowski-Grüss type inequalities," Adv. Appl. Math. Anal., vol. 3, no. 1, pp. 17-20, 2008.

Authors' addresses

Qiaoling Xue

College of Mathematics and Physics, Nanjing University of Information Science and Technology, Nanjing 210044, China

E-mail address: qlx_1@yahoo.com.cn

Shunfeng Wang

College of Mathematics and Physics, Nanjing University of Information Science and Technology, Nanjing 210044, China

E-mail address: wsfnuist@yahoo.com.cn

\section{Wenjun Liu}

College of Mathematics and Physics, Nanjing University of Information Science and Technology, Nanjing 210044, China

E-mail address: wjliuenuist.edu.cn 